\title{
Integrative genomic analyses on interferon- $\lambda s$ and their roles in cancer prediction
}

\author{
LIMING YANG ${ }^{1}$, JIFU WEI $^{1,2}$ and SHAOHENG HE ${ }^{2}$ \\ ${ }^{1}$ School of Life Sciences, Huaiyin Normal University, Huaian, Jiangsu 223300; ${ }^{2}$ Clinical Research Center, \\ the First Affiliated Hospital of Nanjing Medical University, Nanjing, Jiangsu 210029, P.R. China
}

Received September 25, 2009; Accepted November 27, 2009

DOI: 10.3892/ijmm_00000345

\begin{abstract}
Interferon (IFN)- $\lambda \mathrm{s}$, including IFN $-\lambda 1$, IFN $-\lambda 2$, and IFN- $\lambda 3$, are a newly described group of cytokines distantly related to the type I IFNs and IL-10 family members. Besides the antiviral activity, IFN- $\lambda$ s were reported to inhibit various tumor growths in vitro and in vivo. Herein, we identified IFN- $\lambda$ genes from the genome sequences of the human, chimpanzee, macaque, orangutan, mouse, rat and dog, and found that the locations and copy of a specific IFN- $\lambda$ varied in different genomes, not just the copy of IFN- $\lambda$ s. We found human IFN- $\lambda$ s were expressed in fetal retina, fetal brain and T cells by ESTs search. Moreover, IFN- $\lambda$ s were also found to express in bladder cancer, blood cancer, breast cancer, glioma, head and neck cancer and lung cancer tissues. Three tumorrelated transcriptional factors (steroidogenic factor-1, Wilms tumor 1 and P53) binding sites were identified within the $1.0-\mathrm{kb}$ regions upstream of the transcriptional start site of human IFN- $\lambda$ s. Meta-analysis of the prognostic value of IFN- $\lambda$ genes in various cancers showed that the expression of IFN- $\lambda$ s are indeed related to the cancer prognosis in certain types of cancer. It can be predicted that IFN- $\lambda$ s take part in the cancer development by the regulation of expression of IFN- $\lambda$ s related to the SF-1, P53 and WT-1.
\end{abstract}

\section{Introduction}

Interferon (IFN)- $\lambda$ s including IFN- $\lambda 1$, IFN- $\lambda 2$, and IFN- $\lambda 3$, also known as IL-29, IL-28A, or IL-28B, are a newly described

Correspondence to: Professor Shaoheng He, Clinical Research Center, the First Affiliated Hospital of Nanjing Medical University, 300 Guangzhou Road, Nanjing 210029, P.R. China E-mail: shoahenghe@hotmail.com

Dr Jifu Wei, School of Life Sciences, Huaiyin Normal University, Huaian, Jiangsu 223300, P.R. China

E-mail: weijifu@hotmail.com

Key words: interferon- $\lambda$, comparative genomics, comparative proteomics, cancer, meta analysis, P53, SF-1, WT-1 group of cytokines distantly related to the type I IFNs and IL-10 family members. Sheppard et al (1) mapped the IFN- $\lambda 1$, IFN- $\lambda 2$, and IFN- $\lambda 3$ genes in chromosome $19 q 13.13$. The gene localization appeared distinct from that of the related type I IFNs, which are co-localized on chromosome 9. Similar to the IL-10 gene family, IFN- $\lambda$ s contain multiple exons, IFN- $\lambda 2$ and IFN- $\lambda 3$ have six, and IFN- $\lambda 1$ has five exons. In contrast, type I IFNs are encoded within a single exon. The deduced IFN- $\lambda 1$ protein contains 200 amino acids, which is $81 \%$ identical to IFN- $\lambda 2$ and IFN- $\lambda 3$, but it shares only low homology with IL-10, IFN-A2, and IL-22. IFN- $\lambda$ s have a conserved cysteine pattern and amphipathic profile similar to other helical cytokine family members.

It is well known that IFN- $\lambda$ s have antiviral activity against a broad spectrum of viruses including human immunodeficiency virus type 1 (HIV-1) (2), influenza A (3-4), Apeu virus (5), hepatitis C virus (HCV) (6-7), hepatitis B virus (HBV) (8), respiratory syncytial virus (RSV) (9), encephalomyocarditis virus (EMCV) (10) and West Nile virus (11). Another important function of IFN- $\lambda \mathrm{s}$ is their potential antiproliferative activities. IFN- $\lambda \mathrm{s}$ were reported to inhibit proliferation of human glioblastoma LN319 cell line (12), human neuroendocrine BON1 tumor cells (13), human keratinocyte cell line $\mathrm{HaCaT}$ (14), human fibrosarcoma 2fTGH cell line (15) and murine BW5147 thymoma cell line (15) in vitro.

Fox et al used gene structure analysis and comparative genomics to identify IFN- $\lambda$ s from other mammalian genome sequences and found that there is one to potentially nine copies of IFN- $\lambda$ genes in each genome (16). IFN- $\lambda$ s can be induced in various cell lines and primary cells by dsRNA or viral infection $(10,17,18)$. It is hypothesized that IFN- $\lambda$ s and type I IFN genes are regulated by a common mechanism. The IFN- $\lambda 1$ is regulated by virus-activated IFN regulatory factor (IRF) 3 and IRF7, resembling the regulation of the IFN- $\beta$. IFN- $\lambda 2 / 3$ expression is mainly controlled by IRF7, resembling the regulation of IFN- $\alpha$ (19).

In the present study, we identified IFN- $\lambda$ genes from the genome sequences of the human, chimpanzee, macaque, orangutan, mouse, rat and dog by comparative genomic analyses. Conserved transcription factor-binding sites within promoter regions of human IFN- $\lambda$ genes were then searched. Furthermore, meta-analysis of the prognostic value of IFN- $\lambda$ genes in various cancers was also performed. 


\section{Materials and methods}

Identification of novel IFN- $\lambda$ s in mammals and comparative genomic analyses. IFN- $\lambda$ genes were searched in the genome sequences of human (Homo sapiens), chimpanzee (Pan troglodytes), macaque (Macaca mulatta), orangutan (Pongo pygmaeus), mouse (Mus musculus), horse, rat (Rattus norvegicus) and dog (Canis familiaris) using human and mouse IFN- $\lambda s$ as queries. The assemblies used were human NCBI 36, chimpanzee CHIMP2.1, macaque MMUL 1.0, orangutan PPYG2, mouse NCBI m37, rat RGSC 3.4 and dog Canfam 2.0. The identified putative IFN- $\lambda$ genes were BLASTed against the $\mathrm{nr}$ database of GenBank to confirm that the best hits were IFN- $\lambda$ genes. Conserved transcription factor-binding sites within promoter regions of human IFN- $\lambda$ genes, IL-10 and IFN- $\gamma$ were then searched by using the Patch program (http:// www.gene-regulation.com) as well as manual inspection as previously described (20-31).

Comparative proteomic analyses. Amino-acid sequences of the identified IFN- $\lambda$ genes were used for the phylogenetic analysis. The phylogenetic tree of IFN- $\lambda$ genes were obtained by using ML (maximum likelihood) (PHYML v2.4.4) (32) and NJ (neighbor-joining) (MEGA 3.0) (33) methods, and the reliability was evaluated by the bootstrap method with 1,000 replications.

In silico expression analyses. Expressed sequence tags (ESTs) derived from human IFN- $\lambda$ s, IL-10 and IFN- $\gamma$ were searched by using the BLAST programs as previously described (21-31). Human IFN- $\lambda 1$ (NM_172140), IFN- $\lambda 2$ (NP_742150), IFN- $\lambda 3$ (NM_172139), IL-10 (NM_000572) and IFN- $\gamma$ (NM_000619) were used as query sequences for the BLAST programs.

Meta-analysis of the prognostic value of IFN- $\lambda, I L-10$ and $I F N-\gamma$ genes in cancer. A database named 'PrognoScan' has been developed recently (34), which contains a large collection of publicly available cancer microarray datasets with clinical annotation. When a gene is given, PrognoScan displays a summary of tests in a table format for the gene. The parameters include dataset, cancer type, subtype, endpoint, cohort, contributor, array type, probe ID, number of patient, optimal cutpoint, $P$ min and $P$ cor. It can also be used as a tool for assessing the biological relationship between gene expression and cancer prognosis. PrognoScan employs the minimum $\mathrm{P}$-value approach for grouping patients for survival analysis. It provides a powerful platform for evaluating potential tumor markers and therapeutic targets, and is accessible at http://gibk21.bse.kyutech.ac.jp/PrognoScan/index.html. Human IFN- $\lambda$, IL-10 and IFN- $\gamma$ genes were inputted as queries and the data were collected for further analysis.

\section{Results}

Identification of IFN- $\lambda$ s in mammal genomes. IFN- $\lambda$ genes were searched in the genome sequences of human, chimpanzee, macaque, orangutan, mouse, rat and dog. While three IFN $-\lambda$ genes were identified from human, dog, orangutan genomes, only two IFN- $\lambda$ genes were identified in macaque and mouse genomes. Interestingly, rat has only one IFN- $\lambda$ gene in its



Figure 1. Phylogenetic analysis of IFN- $\lambda \mathrm{s}$. The phylogenetic tree of IFN- $\lambda$ gene was obtained by using maximum likelihood and neighbor-joining methods. It seemed that IFN- $\lambda$ distinctly separated from IFN- $\lambda 2$ and IFN- $\lambda 3$.

genome, whereas horse possesses 4 IFN- $\lambda$ genes in its genome. For chimpanzee genome, three IFN- $\lambda$ genes were identified, but only one complete IFN- $\lambda$ sequence was obtained. The other two sequences were not available due to the uncompleted genome sequencing project. The amino acid sequences of the identified IFN- $\lambda$ s can be obtained upon request.

Comparative proteomics on mammalian IFN- $\lambda$ genes. Refined phylogenetic tree using the identified IFN- $\lambda$ amino acid sequences by ML and NJ methods was almost identical. IFN- $\lambda 1 \mathrm{~s}$ were clustered into one group and distinctly separated from IFN- $\lambda 2$ and IFN- $\lambda 3$ (Fig. 1). However, IFN- $\lambda 2$ and IFN- $\lambda 3$ were clustered into another group and not separated from each other.

Expression profile of human IFN- $\lambda$ s. Human IFN- $\lambda 1$ was expressed in fetal brain and $\mathrm{T}$ cells whereas human IFN- $\lambda 2$ and $-\lambda 3$ were expressed in fetal retina, brain and $\mathrm{T}$ cells. When searched in PrognoScan database, human IFN- $\lambda$ s were also found to be highly expressed in bladder cancer, blood cancer, breast cancer, glioma, head and neck cancer and lung cancer tissues. IL-10 was expressed in blood, spleen, lung con-nective tissue and T cells. IFN- $\lambda$ was expressed in blood, kidney, lymph node and fetal brain.

Comparative genomics on human IFN- $\lambda s$, IL-10 and IFN- $\gamma$. Transcription factor-binding sites within the 5'-region of human IFN- $\lambda \mathrm{s}$ were identified. Within the $1.0-\mathrm{kb}$ regions 
Table I. Meta-analysis of the prognostic value of IFN- $\lambda$ s, IL-10 and IFN- $\gamma$ genes in cancer.

\begin{tabular}{lcccccc}
\hline & \multicolumn{6}{c}{ Cancers } \\
\cline { 2 - 7 } Genes & Bladder & Blood & Breast & Glioma & $\begin{array}{c}\text { Head and } \\
\text { neck }\end{array}$ & Lung \\
\hline IFN- $\lambda 1$ & $0 / 1$ & $0 / 2$ & $2 / 4$ & $0 / 1$ & $0 / 1$ & $0 / 2$ \\
IFN- $\lambda 2$ & $0 / 1$ & $0 / 2$ & $1 / 4$ & $0 / 1$ & $0 / 1$ & $0 / 2$ \\
IFN- $\lambda 3$ & $1 / 1$ & $1 / 2$ & $1 / 4$ & $0 / 1$ & $0 / 3$ & $0 / 2$ \\
IFN- $\gamma$ & $0 / 2$ & $0 / 7$ & $2 / 29$ & $0 / 2$ & $0 / 1$ & $0 / 8$ \\
IL-10 & $0 / 2$ & $0 / 7$ & $3 / 25$ & $0 / 2$ & $0 / 1$ & $0 / 6$ \\
\hline
\end{tabular}

Data were expressed as $\mathrm{n} / \mathrm{m}$. n represents the test number that showed an association between microarray expression of genes and cancer prognosis with $5 \%$ significance level. $\mathrm{m}$ represents the test number.

upstream of the transcriptional start sites, four steroidogenic factor-1 (SF-1), three Wilms tumor 1 (WT1) and one P53 binding sites were identified for human IFN- $\lambda 1$; three SF-1, four WT-1 and two P53 binding sites were identified for human IFN- $\lambda 2$; five WT-1 and two P53 binding sites was identified for human IFN- $\lambda 3$. Two SF-1, WT-1 and P53 binding sites were identified for human IL-10. Only one SF-1 and WT-1 and P53 binding sites were identified for human IFN- $\gamma$ (Fig. 2)

Meta-analysis of the prognostic value of IFN- $\lambda, I F N-\gamma$ and $I L-10$ genes in cancer. Using the databases detecting the expression of IFN- $\lambda \mathrm{s}, 2$ out of 11 tests showed an association between microarray expression of IFN- $\lambda 1$ and cancer prognosis with $5 \%$ significance level. By clicking the probe ID of these positive breast cancer cases in the list, we found the Rotterdam cohort for distant metastasis-free survival (DMFS) and recurrence-free survival (RFS) that patients can be dichotomized at the 10 percentile to give the minimum $\mathrm{P}$-value and the group with low IFN- $\lambda 1$ expression has poorer survival chance (Pcor $=0.002)$ (Table I). Moreover, these two cases showed no association between expression in IFN- $\lambda 2$ and IFN- $\lambda 3$ with cancer prognosis. One out of 11 tests showed an association between microarray expression in IFN- $\lambda 2$ and cancer prognosis with 5\% significance level. Rotterdam cohort for RFS that patients can be dichotomized at the 84 percentile to give the minimum $\mathrm{P}$-value and the group with high IFN- $\lambda 2$ expression has poorer survival chance $(P$ cor $=0.0087)$ in this breast cancer case (Table I). Three out of 13 tests showed an association between microarray expression in IFN- $\lambda 3$ and cancer prognosis with $5 \%$ significance level. In the bladder cancer case, Rotterdam cohort for overall survival (OS) that patients can be dichotomized at the 33 percentile to give the minimum P-value and the group with high IFN- $\lambda 3$ expression has poorer survival chance $(P$ cor $=0.0366)$.

In the blood cancer (multiple myeloma) case, Rotterdam cohort for Arkansas that patients can be dichotomized at the 20 percentile to give the minimum $\mathrm{P}$-value and the group with low IFN- $\lambda 3$ expression has poorer survival chance
(Pcor $=0.0071)$. In the breast cancer case, Rotterdam cohort for RFS that patients can be dichotomized at the 88 percentile to give the minimum P-value and the group with high IFN- $\lambda 3$ expression has poorer survival chance $(P$ cor $=0.0066)$, very similar to the result of IFN- $\lambda 2$ (Table I). Using the databases detecting the expression of IFN- $\gamma$ and IL-10, 2 out of 49 tests and 3 out of 43 tests showed an association between microarray expression of IFN- $\gamma$ and IL-10 with cancer prognosis, respectively (Table I).

\section{Discussion}

By comparative genomic analysis, we found that IFN $-\lambda 1$, IFN- $\lambda 2$ and IFN- $\lambda 3$ genes exist in two primate (human and orangutan) genomes. However, only IFN- $\lambda 2$, and IFN- $\lambda 3$ genes were found in the other primate (macaque) genome. In dog genome, two IFN- $\lambda 1 \mathrm{~s}$ are clustered in the chromosome 1, whereas the IFN- $\lambda 2$ is located in chromosome 24 . However, two IFN- $\lambda 1 \mathrm{~s}$ are identified in chromosomes 10 and 22 in horse genome, whereas IFN- $\lambda 2$ and IFN- $\lambda 3$ genes are found only in chromosomes 10. It seemed that the locations and copy of specific IFN- $\lambda$ s varied in different genomes, not just the copy of IFN- $\lambda$ s. It may be due to the gene conversion and gene duplication, which have shaped the evolution of the IFN- $\lambda$ gene family in eutherian species. It is similar to the evolution of IFN- $\alpha$, which consists of a 13 member multigene family in human genome (35). Members of the IFN- $\alpha$ gene family in different species normally reside in close proximity within a single chromosome. Phylogenetically, IFN- $\alpha$ family members in eutherians (placental mammals) cluster together in a speciesspecific manner except for closely related species (i.e. Homo sapiens and Pan troglodytes) and formed due to both gene conversion and gene duplication (36).

IFN- $\lambda$ expression was detected in human blood, brain, lung, ovary, pancreas, pituitary, placenta, prostate, and testis (1). They are also induced in various cell lines and primary cells by dsRNA or viral infection $(1,14)$. In the present study, we found human IFN- $\lambda$ s were also expressed in fetal retina, fetal brain and $\mathrm{T}$ cells by ESTs search, indicating that IFN- $\lambda$ s are early products of human life, which most likely participate in the innate immunity in early life. Other type I IFNs (IFN- $\gamma$ ) and IL-10 family members (IL-10) showed different expression patterns with IFN- $\lambda$ s. Moreover, IFN- $\lambda$ s were also found to express in bladder cancer, blood cancer, breast cancer, glioma, head and neck cancer and lung cancer tissues. IFN- $\lambda$ and IL-10 were also found to express in these cancers.

Former study found that IFN- $\lambda 1$ and IFN- $\lambda 3$ gene promoters had functional IFN-stimulated response element and NF-kappaB binding sites and that the IFN- $\lambda 1$ gene is regulated by virus-activated IRF3 and IRF7, whereas IFN- $\lambda 2$ and IFN- $\lambda 3$ gene expression is mainly controlled by IRF7 (19). In the present study, we identified different numbers of SF-1, WT1 and P53 binding sites located within the $1.0-\mathrm{kb}$ regions upstream of the transcriptional start site of human IFN- $\lambda 1$, 1234 IFN- $\lambda 2$ and IFN- $\lambda 3$ genes, though the amino acid sequences of human IFN- $\lambda 2$ and IFN- $\lambda 3$ showed high similarity. The IFN- $\lambda 1$ and IFN- $\lambda 3$ gene promoters were different at least in the SF-1 binding sites. It suggested that 


\begin{abstract}
TTAACCAGTCAAGGTGACACCTAAAATTAACCATCACAATTATAAAAATAACTACTCAGAGAAACATTAGGA GCATGAACTGAAATTAGTTAATGGGACATTCTTAAACCAATGGCAGAAGCTCCTTCTTGGCCAGGAGCAGTG GCTCATGCCTTTAATACTAGCACTTTGCGAGGCTGAAGCAGGAGGATGGCTTAAGGCCAGGAGTTCAAGACT GGCCTGGGCAACATAGTGAGACCCCTATCTCTACAAAAATAAATAAATAAATAATAAAGTAAGGTGGTGGCT CACGCCTGTAATCCCAGCACTTTGGGAGGCCAAGGCAGGCAGATCATCTGAAGTCAGGAGTTCGAAGCCAGC GTGACCAACATAGTAAAACCCAGTCTCTACTAAAAATACAAAAACTAGCCAGGCGTGATGGCATGCACCTGT AATCCCAACTACTTAGGAGGCTGAGGCAGGAGAATCGCTTCAACTCGGGAGGCAGAAGTTGCAGTGAGCCAA GATTGCACCATTGCACTCCAGCCTGGGCAACAAGAGCAAAACTACGTCTCAAAAAATAATAATAACAATAAA ATAAAAAACAAGCTTTTTTTTTTTTGAAACAGGATCTCACTCCATCACCCAGGCTGGAGTGCAGTGGCACGA TCTTGGCTCACTGCAACCTCCGCCTCCCGGGTTCAAGTGATTCTCATGCCTCGGCCTCCTGAGTAGCTGAGA CCACAGGCGCATGCCACCACACCTGGCTAATTTAGAATAAAAAAGAAGCTTCCTCTCTGCCACTCAGGTAGC CTTATCCCTAATCTCAGCCTCCGTCAGGGACTCCCTGAGGCCAGTTGGCTGAAAGCTGCCCAGGGAGTTCTA AGGATTTCAGTTTCTCTTTCCTTCTTGATGCAGCTCCCAGCTCACTTGGCCCTGCCCACACCTGTTCCCTCA TCAGGCTCCCAGACGGGCCCCGCCCACTCATGCCTCTTAAGTCAAAGTGGAAATTCTCATTTCCAATTACCT TTTCACTTTACACACATCATCTTGGATTGCCCATTTTGCGTGGCTAAAAAGCAGAGCCATGCCGCTGGGGAA GCAGTTGCGATTTAGCCATG
\end{abstract}

\title{
B. IFN- $\lambda 2$
}

TGGGCGTGGTGGTGCATGCCTATAGTCCTTTCTACTCGGGAGGCTGAAGCAGAAGAATCCCTTGAACCAAGG AGGTGGAGGTTGCAGTGAGTCGAGATCACGCTGCTGCACTCCAGCCTGGGCTACAGAGCAAGACTCCATCTC AAAAAAAAAAGAAAAAAGAAAGAAAGAAAGAAATCATGGCCTCTGGGCACAGTGGCTCATGCCTGCAACCCC AGCAATTTGGGAGGCCAAGACAGACAGATCACTTGACGTCAAGAGTTCGAGACCAGCCTGGCCAATTGGTGA ACTGTCATCTCTACTAAAACCATAAAAATTAGCTGGGAATGGTGGCACAAATCTGTAATCTCAGCTACTTGG GAGGCTAAGGCAAGAGAATCGCTTGAACCCAGGAGGTGGAGGTTGCAGTTAGTCAAGATTTTGCACTGCACT CCAGCCTGGGTGACCGAACAAGACCCTGTCTCAAAATATATATATATATATATGCCAGGAGGGTGGCTCAG GCCTGTAATCTCAGCACTTTAATAGGCTGGGTGAGGAGGATGGCTTGAGCCCAGGAGTTTGAGGCTGCAGTG AGCTGTGATCATGCCATTGCACTCCAGTGACAGAGTGAGACCCTGTCTTAAACAACAACAAAACCAGAGCAG GTGGAATCCTCCTGGGAACATACCTTCCTGTAGGTTACCCCTGAGTCTCCATCAGTTTCTCTTTCCCTCCAG CTGCTCATCTGGCTCACTAGCCCTGCCCTGCTCTGGGCTTTCCCAGCCTGGGGCTCCCCTGGTGGCCGGTGT CTTACCTGAGGCTGTGTTTTCACTTTTCCTACATCAGCTGGGACTGCCCTTCTGTCAGGGATAAAAGCTGCC CCATGGAGCTCAGGCAGGAATTACATCCCAGACAGAGCTCAAAACTGACAGAAAGAGTCAAAGCCAGGACAC AGTCTGAGATCCAGAAGAGGGGACTGAAAAGAACAGAGACTCCAGACAAGACCCAAACAGACCCTGGGTGAC AGCCTCAGAGTGTTTCTTCTGCTGACAAAGACCAGAGATCAGGAATG

\section{IFN- $\lambda 3$}

TTTGAACCCCAGACCCTTCATCTCCAAGACCCATGCTCTTCACCACTGCCTGAACTTCCCTAAGAAAGACGG CACCCACGTGGTGTCCTTCAAGTCCTTCGTCACACCTCAATTCTTGAGCAGAGCCTCATATTCCTGAGTCCT TCCTTGCCTGGGCAATTAAGAAATATTGGCCTCTGGGCATGGTGGCTCACACTGAAATCCCAGCAATTTGGG AGGCCTAGACAGAGAGATGACTTGACATCAGGAATTTGAGACCAGCCTTGCCAACATGGTGAAACGCCATCT CTACTAAAAATATAAAAATTAGCTGGGAATGGTGGCACAAATCTGTAATCTCAGCTACTTGGGAGGCTAAGG CAAGAGAATTGCTTGAACCCAGGAGGCGGAGGTTGCAGTTAGCCAAGATTTTGCACTGCACTCCAGCCTGGG TGACCGAACAAGACCCTGTCTCAAAATATATATATATATATATATATATATATGCCAGGAGTGGTGGCTCAG GCCTGTAATCTCAGCACTTTAATAGGCTGGGTGAGGAGGATGGCTTGAGCCCAGGAGTTTGAGGCTGCAGTG AGCTGTGATCATGCCATTGCACTGCAGTGACAGAGTGAGACCCTGTCTTAAACAACAACAAAACCAGAGCAG GTGGAATCCTCTTGGGAACATACCTTCCTGTAGGTTACCCCTGAGTCTCCATCAGTTTCTCTTTCCCTCCAG CTGCTCATCTGGCTCACTAGCCCTGCCCTGCTCTGGGCTTTCCCAGCCTGGGGCTCCCCTGGTGGCCGGTGT CTTACCTGAGGCTGTGTTTTCACTTTTCCTACATCAGCTGGGACTGCCCTTCTGTCAGGGATAAAAGCTGCC CCATGGAGCTCAGGCAGGAATTACATCCCAGACAGAGCTCAAAACTGACAGAAAGAGTCAAAGCCAGGACAC AGTCTGAGATCCAGAAGAGGGGACTGAAAAGAACAGAGACTCCAGACAAGACCCAAACAGACCCTGGGTGAC AGCCTCAGAGTGTTTCTTCTGCTGACAAAGACCAGAGATCAGGAATGAAACTAGGTGAGTCCCACATCTCTG TCCGTGCTCAGCTCCTGCAGCCCCTGCCCTCAGTGGGCAGCCTCTGCATTCCCTCAGCTCCCTTTCTCTCTG TGACACAGACATG

Figure 2. The identification of transcription factor-binding sites within the 5'-region of human IFN- $\lambda 1$ (A), IFN- $\lambda 2$ (B), IFN- $\lambda 3$ (C).

human IFN- $\lambda 2$ and IFN- $\lambda 3$ play different roles in their function due to different expression models. Moreover, IFN- $\lambda \mathrm{s}$ showed different promoter structures with IFN- $\gamma$ and IL-10.

WT1 is a zinc finger DNA-binding protein and acts as a transcriptional activator or repressor depending on the cellular or chromosomal context. The WT1 gene is overexpressed in leukemias and various types of solid tumors, and the WT1 protein was demonstrated to be an attractive target antigen for immunotherapy against these malignancies (37). The p53 gene is mutated in about half of all human tumors. p53 is a 


\begin{abstract}
D. IL-10
TTATTTCAACTTCTTCCACCCCATCTTTTAAACTTTAGACTCCAGCCACAGAAGCTTACAACTAAAAGA AACTCTAAGGCCAATTTAATCCAAGGTTTCATTCTATGTGCTGGAGATGGTGTACAGTAGGGTGAGGAA ACCAAATTCTCAGTTGGCACTGGTGTACCCTTGTACAGGTGATGTAATATCTCTGTGCCTCAGTTTGCT CACTATAAAATAGAGACGGTAGGGGTCATGGTGAGCACTACCTGACTAGCATATAAGAAGCTTTCAGCA AGTGCAGACTACTCTTACCCACTTCCCCCAAGCACAGTTGGGGTGGGGACAGCTGAAGAGGTGGAAAC ATGTGCCTGAGAATCCTAATGAAATCGGGGTAAAGGAGCCTGGAACACATCCTGTGACCCCGCCTGTAC TGTAGGAAGCCAGTCTCTGGAAAGTAAAATGGAAGGGCTGCTTGGGAACTTTGAGGATATTTAGCCCAC CCCCTCATTTTTACTTGGGGAAACTAAGGCCCAGAGACCTAAGGTGACTGCCTAAGTTAGCAAGGAGAA GTCTTGGGTATTCATCCCAGGTTGGGGGGACCCAATTATTTCTCAATCCCATTGTATTCTGGAATGGGC AATTTGTCCACGTCACTGTGACCTAGGAACACGCGAATGAGAACCCACAGCTGAGGGCCTCTGCGCACA GAACAGCTGTTCTCCCCAGGAAATCAACTTTTTTTAATTGAGAAGCTAAAAAATTATTCTAAGAGAGGT AGCCCATCCTAAAAATAGCTGTAATGCAGAAGTTCATGTTCAACCAATCATTTTTGCTTACGATGCAAA AATTGAAAACTAAGTTTATTAGAGAGGTTAGAGAAGGAGGAGCTCTAAGCAGAAAAAATCCTGTGCCGG GAAACCTTGATTGTGGCTTTTTAATGAATGAAGAGGCCTCCCTGAGCTTACAATATAAAAGGGGGACAG AGAGGTGAAGGTCTACACATCAGGGGCTTGCTCTTGCAAAACCAAACCACAAGACAGACTTGCAAAAGA AGGCATG
\end{abstract}

\section{E. IFN- $\gamma$}

CCACCTTCTGGGTTCAAGCCATTCTCCTGCCTCAGCCTCCCTAGTAGCTGAGATTACAGGCATACACCA CCATGCCTGGCTAATTTTTTTGTATTTTTAGTAGAGATGGGGTTTCACCATATTGGCTAGGCTGGTCTC AAACTCCTGACCTTGTGATCCACCCGCCTCAACCTCCCAAAGTGCTGGGATTACAGGTGTGAGCCACTG CGTCTGGAACTCCCCCTGGGAATATTCTCTACACTGTATTTCAAGGATTTAATATGACAAAAAGAATGT CAAATACCTTATTAACAATGTAGTATATTGATGCATACTGAAGTACTATTTGGGATATATTGGTTTAAA TACAATATATTTTAAAATTATATTTACCTTTTAAAAAAACTTTTATTAATGAGGCTACTAGATCATTTA AATTTACCTGTGTGGCTTGTATTGTATTTCTACTGGGCAGTGCTGATCTAGAGCAATTTGAAACTTGTG GTAGATATTTTACTAACCAACTCTGATGAAGGACTTCCTCACCAAATTGTTCTTTTAACCGCATTCTTT CCTTGCTTTCTGGTCATTTGCAAGAAAAATTTTAAAAGGCTGCCCCTTTGTAAAGGTTTGAGAGGCCCT AGAATTTCGTTTTTCACTTGTTCCCAACCACAAGCAAATGATCAATGTGCTTTGTGAATGAAGAGTCAA CATTTTACCAGGGCGAAGTGGGGAGGTACAAAAAAATTTCCAGTCCTTGAATGGTGTGAAGTAAAAGTG CCTTCAAAGAATCCCACCAGAATGGCACAGGTGGGCATAATGGGTCTGTCTCATCGTCAAAGGACCCAA GGAGTCTAAAGGAAACTCTAACTACAACACCCAAATGCCACAAAACCTTAGTTATTAATACAAACTATC ATCCCTGCCTATCTGTCACCATCTCATCTTAAAAAACTTGTGAAAATACGTAATCCTCAGGAGACTTCA ATTAGGTATAAATACCAGCAGCCAGAGGAGGTGCAGCACATTGTTCTGATCATCTGAAGATCAGCTATT AGAAGAGAAAGATCAGTTAAGTCCTTTGGACCTGATCAGCTTGATACAAGAACTACTGATTTCAACTTC TTTGGCTTAATTCTCTCGGAAACGATG

Figure 2. The identification of transcription factor-binding sites within the 5'-region of human IL-10 (D) and IFN- $\gamma$ (E). SF-1-binding sites (underline), WT-1binding sites (double underline), and P53-binding sites (dotted line) are indicated. The transcriptional start sites (ATG) of human IFN- $\lambda$ s, IL-10 and IFN- $\gamma$ are indicated in bold.

transcription factor whose activity gives rise to a variety of cellular outcomes, most notably cell cycle arrest and apoptosis, eliminating cancer-prone cells from the replicative pool (38). SF-1 is an orphan nuclear receptor that regulates the transcription of an array of genes involved in reproduction, steroidogenesis, and male sexual differentiation. SF-1 amplification and overexpression are found in various tumors (39-41). IFN- $\lambda \mathrm{s}$ were found to inhibit tumor growth in vitro and in vivo (12-15). However, the detailed role in the development of cancer was still unknown.

Meta-analysis of the prognostic value of IFN- $\lambda$ genes in various cancers found that the expression of IFN- $\lambda$ is indeed related to the cancer prognosis in certain types of cancer. Moreover, the expression of human IFN- $\lambda 2$ and IFN- $\lambda 3$ was differently related to the cancer prognosis. The expression of IFN- $\lambda$ s related to the cancer prognosis is different from other type I IFNs (IFN- $\gamma$ ) and IL-10 family members (IL-10). By combining the identifications of transcription factor-binding sites related to tumors in the promoter regions of IFN- $\lambda \mathrm{s}$, it can be predicted that IFN- $\lambda$ s take part in cancer development by the the regulation of expression of IFN- $\lambda$ s related to the SF-1, P53 and WT-1.

\section{References}

1. Sheppard P, Kindsvogel W, Xu W, et al: IL-28, IL-29 and their class II cytokine receptor IL-28R. Nat Immunol 1: 63-82, 2003.

2. Hou W, Wang X, Ye L, et al: Lambda interferon inhibits human immunodeficiency virus type 1 infection of macrophages. J Virol 83: 3834-3842, 2009.

3. Wang J, Oberley-Deegan R, Wang S, et al: Differentiated human alveolar type II cells secrete antiviral IL-29 (IFNlambda1) in response to influenza A infection. J Immunol 182: 1296-1304, 2009.

4. Hui KP, Lee SM, Cheung CY, et al: Induction of proinflammatory cytokines in primary human macrophages by influenza A virus (H5N1) is selectively regulated by IFN regulatory factor 3 and p38 MAPK. J Immunol 182: 1088-1098, 2009. 
5. Almeida GM, de Oliveira DB, Magalhães CL, Bonjardim CA, Ferreira PC and Kroon EG: Antiviral activity of type I interferons and interleukins 29 and 28a (type III interferons) against Apeu virus. Antiviral Res 80: 302-308, 2008.

6. Pagliaccetti NE, Eduardo R, Kleinstein SH, Mu XJ, Bandi P and Robek MD: Interleukin-29 functions cooperatively with interferon to induce antiviral gene expression and inhibit hepatitis C virus replication. J Biol Chem 283: 30079-30089, 2008.

7. Marcello T, Grakoui A, Barba-Spaeth G, et al: Interferons alpha and lambda inhibit hepatitis $C$ virus replication with distinct signal transduction and gene regulation kinetics. Gastroenterology 131: 1887-1898, 2006.

8. Hong SH, Cho O, Kim K, Shin HJ, Kotenko SV and Park S: Effect of interferon-lambda on replication of hepatitis B virus in human hepatoma cells. Virus Res 126: 245-249, 2007.

9. Chi B, Dickensheets HL, Spann KM, et al: Alpha and lambda interferon together mediate suppression of $\mathrm{CD} 4 \mathrm{~T}$ cells induced by respiratory syncytial virus. J Virol 80: 5032-5040, 2006.

10. Brand S, Beigel F, Olszak T, et al: IL-28A and IL-29 mediate antiproliferative and antiviral signals in intestinal epithelial cells and murine CMV infection increases colonic IL-28A expression. Am J Physiol Gastrointest Liver Physiol 289: G960-G968, 2005.

11. Ma D, Jiang D, Qing M, et al: Antiviral effect of interferon lambda against West Nile virus. Antiviral Res 83: 53-60, 2009.

12. Meager A, Visvalingam K, Dilger P, Bryan D and Wadhwa M: Biological activity of interleukins-28 and -29: comparison with type I interferons. Cytokine 31: 109-118, 2005.

13. Zitzmann K, Brand S, Baehs S, et al: Novel interferon-lambdas induce antiproliferative effects in neuroendocrine tumor cells. Biochem Biophys Res Commun 344: 1334-1341, 2006.

14. Maher SG, Sheikh F, Scarzello AJ, et al: IFNalpha and IFNlambda differ in their antiproliferative effects and duration of JAK/STAT signaling activity. Cancer Biol Ther 7: 1109-1115, 2008.

15. Dumoutier L, Tounsi A, Michiels T, Sommereyns C, Kotenko SV and Renauld JC: Role of the interleukin (IL)-28 receptor tyrosine residues for antiviral and antiproliferative activity of IL-29/ interferon-lambda 1: similarities with type I interferon signaling. J Biol Chem 279: 32269-32274, 2004.

16. Fox BA, Sheppard PO and O'Hara PJ: The role of genomic data in the discovery, annotation and evolutionary interpretation of the interferon-lambda family. PLoS One 4: E4933, 2009.

17. Osterlund P, Veckman V, Sirén J, et al: Gene expression and antiviral activity of alpha/beta interferons and interleukin-29 in virus-infected human myeloid dendritic cells. J Virol 79: 9608-9617, 2005.

18. Melchjorsen J, Sirén J, Julkunen I, Paludan SR and Matikainen S: Induction of cytokine expression by herpes simplex virus in human monocyte-derived macrophages and dendritic cells is dependent on virus replication and is counteracted by ICP 27 targeting NF-kappaB and IRF-3. J Gen Virol 87: 1099-1108, 2006.

19. Osterlund PI, Pietilä TE, Veckman V, Kotenko SV and Julkunen I: IFN regulatory factor family members differentially regulate the expression of type III IFN (IFN-lambda) genes. J Immunol 179: 3434-3442, 2007.

20. Katoh Y and Katoh M: Integrative genomic analyses on GLI1: positive regulation of GLI1 by Hedgehog-GLI, TGFbeta-Smads, and RTK-PI3K-AKT signals, and negative regulation of GLI1 by Notch-CSL-HES/HEY, and GPCR-Gs-PKA signals. Int J Oncol 35: 187-192, 2009
21. Katoh $\mathrm{Y}$ and Katoh M: Integrative genomic analyses on GLI2: mechanism of Hedgehog priming through basal GLI2 expression, and interaction map of stem cell signaling network with P53. Int J Oncol 33: 881-886, 2008.

22. Katoh Y and Katoh M: Comparative integromics on JMJD2A, JMJD2B and JMJD2C: preferential expression of JMJD2C in undifferentiated ES cells. Int J Mol Med 20: 269-273, 2007.

23. Katoh Y and Katoh M: Comparative genomics on PROM1 gene encoding stem cell marker CD133. Int J Mol Med 19: 967-970, 2007.

24. Katoh Y and Katoh M: Conserved POU-binding site linked to SP1-binding site within FZD5 promoter: Transcriptional mechanisms of FZD5 in undifferentiated human ES cells, fetal liver/spleen, adult colon, pancreatic islet, and diffuse-type gastric cancer. Int J Oncol 30: 751-755, 2007.

25. Katoh Y and Katoh M: Comparative integromics on FAT1, FAT2, FAT3 and FAT4. Int J Mol Med 18: 523-528, 2006.

26. Katoh Y and Katoh M: Comparative integromics on VEGF family members. Int J Oncol 28: 1585-1589, 2006.

27. Katoh Y and Katoh M: Comparative integromics on Angiopoietin family members. Int J Mol Med 17: 1145-1149, 2006.

28. Katoh Y and Katoh M: Comparative integromics on BMP/GDF family. Int J Mol Med 17: 951-955, 2006.

29. Katoh Y and Katoh M: Comparative integromics on Ephrin family. Oncol Rep 15: 1391-1395, 2006.

30. Katoh Y and Katoh M: Comparative genomics on HHIP family orthologs. Int J Mol Med 17: 391-395, 2006.

31. Katoh Y and Katoh M: Comparative genomics on SLIT1, SLIT2, and SLIT3 orthologs. Oncol Rep 14: 1351-1355, 2005.

32. Guindon S, Lethiec F, Duroux P and Gascuel O: PHYML Online a web server for fast maximum likelihood-based phylogenetic inference. Nucleic Acids Res 33: W557-W559, 2005.

33. Kumar S, Tamura K and Nei M: MEGA3: Integrated software for molecular evolutionary genetics analysis and sequence alignment. Brief Bioinform 5: 150-163, 2004.

34. Mizuno H, Kitada K, Nakai K and Sarai A: PrognoScan: a new database for meta-analysis of the prognostic value of genes. BMC Med Genomics 2:18, 2009.

35. Pestka S, Krause CD and Walter MR: Interferons, interferonlike cytokines, and their receptors. Immunol Rev 202: 8-32, 2004.

36. Woelk CH, Frost SD, Richman DD, Higley PE and Kosakovsky Pond SL: Evolution of the interferon alpha gene family in eutherian mammals. Gene 397: 38-50. 2007.

37. Hirose M: The role of Wilms' tumor genes. J Med Invest 46: 130-140, 1999.

38. Michael D and Oren M: The p53 and Mdm2 families in cancer. Curr Opin Genet Dev 12: 53-59, 2002.

39. Zhao C, Barner R, Vinh TN, McManus K, Dabbs D and Vang R: SF-1 is a diagnostically useful immunohistochemical marker and comparable to other sex cord-stromal tumor markers for the differential diagnosis of ovarian sertoli cell tumor. Int J Gynecol Pathol 27: 507-514, 2008.

40. Doghman M, Karpova T, Rodrigues GA, et al: Increased steroidogenic factor-1 dosage triggers adrenocortical cell proliferation and cancer. Mol Endocrinol 21: 2968-2987, 2007.

41. Pianovski MA, Cavalli LR, Figueiredo BC, et al: SF-1 overexpression in childhood adrenocortical tumours. Eur J Cancer 42: 1040-1043, 2006. 